\title{
Genetic association between COPD and polymorphisms in TNF, ADRB2 and EPHX1
}

\author{
J. Brøgger*\#, V.M. Steen*,", H.G. Eiken*, ${ }^{*}$, A. Gulsvik" and P. Bakke ${ }^{\#}$
}

\begin{abstract}
There is evidence of a hereditary component in chronic obstructive pulmonary disease (COPD). A number of genetic association studies have been performed to find susceptibility genes of COPD.
\end{abstract}

The current authors performed a case-control, genetic-association study and a meta-analysis of 16 studies, involving seven polymorphisms in three well-studied genes: microsomal epoxide hydroxylase (EPHX1); tumour necrosis factor; and $\beta_{2}$-adrenoreceptor. A total of 492 Caucasian smokers and former smokers were recruited from hospital databases and population cohort studies.

In the present study, a protective effect of the EPHX1 Tyr113His polymorphism was found (homozygous odds ratio (OR) 0.5 ). In the meta-analysis, homozygotes for this single nucleotide polymorphism (SNP) also had a pooled OR of 0.5 . The same effect has been found in several lung cancer studies. Effects for other candidate SNPs were weak or statistically insignificant, and probable genotyping error was common.

In conclusion, the present data and meta-analysis support a role for microsomal epoxide hydroxylase in the aetiology of chronic obstructive pulmonary disease.

KEYWORDS: $\boldsymbol{\beta}_{2}$-adrenergic receptor, case-control study, chronic obstructive pulmonary disease, genetics, microsomal epoxide hydroxylase, tumour necrosis factor- $\alpha$

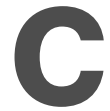
hronic obstructive pulmonary disease (COPD) is a major and increasing cause of morbidity and mortality worldwide [1]. It is characterised by progressive irreversible airflow limitation. The major environmental risk factor is smoking. There is a clear dose-response relationship between lung function and smoking exposure, although there is a substantial heterogeneity in lung function levels [2]. Only a subset of smokers develop an accelerated rate of decline in lung function that leads to COPD. There appears to be a familial clustering of both lung function and COPD $[3,4]$. These insights suggest that susceptibility to COPD may be influenced by genetic factors.

The only well-established genetic cause of COPD, $\alpha_{1}$-antitrypsin deficiency, is rare. A number of studies have been performed to find other genetic susceptibility factors for COPD. Family-based linkage studies with large pedigrees can be used to locate broad genomic regions associated with lung function [5] and early-onset COPD [6, 7]. However, such family-based studies of the more common late-onset COPD are more difficult to perform due to advanced age of the patients at the time of diagnosis. As an alternative approach, a number of genetic association studies have been performed for COPD and its components [8,9]. So far, $>25$ different candidate genes have been tested.

In order to replicate a subset of these previous genetic associations in COPD, a case-control study was performed in 492 Caucasian current or former smokers with and without COPD. The current authors chose single nucleotide polymorphisms (SNPs) in three relatively well-studied interesting candidate genes: tumour necrosis factor (TNF)- $\alpha, \beta_{2}$-adrenoreceptor (ADRB2) and microsomal epoxide hydroxylase (EPHX1). A systematic literature review and meta-analysis of previous studies was also performed.

EPHX1 is an enzyme involved in the detoxification of harmful epoxides from smoking, chemicals and drugs, which is strongly expressed in bronchial epithelium [12]. Enzyme activity varies widely in the population and two common coding polymorphisms confer fast and slow enzyme activity, Tyr113His and His139Arg, respectively [12, 13]. An initial study suggested a strong genetic association with COPD for the
AFFILIATIONS

${ }^{*}$ Center for Medical Genetics and Molecular Medicine, Haukeland University Hospital,

\#Dept of Thoracic Medicine, Institute of Medicine, and

- Section for Medical Genetics and Molecular Medicine, Dept of Clinical Medicine, University of Bergen, Bergen, Norway.

\section{CORRESPONDENCE}

J. Brøgger

Dept of Neurology Haukeland University Hospital Helse Bergen HF

Jonas Liesv. 65

Bergen N-5021

Norway

E-mail: jan.brogger@med.uib.no

Received:

May 152005

Accepted after revision

December 092005

\section{SUPPORT STATEMENT}

The present case-control study was initiated and financed by

GlaxoSmithKline. The genotyping and

DNA extraction was financed

independently by the Western Norway Regional Health Authority and

performed at the Centre for Molecular Medicine (Bergen, Norway). J.

Brøgger took part in the

establishment of the case-control study, wrote the protocol and grant applications, took part in DNA

extraction, performed all genotyping, performed the systematic review and all analyses, and wrote the first and subsequent drafts of this paper. V.M. Steen and H.G. Eiken oversaw DNA extraction, genotyping and developed the paper. A. Gulsvik and P. Bergen took part in the establishment of the case-control studies and developed the paper.

European Respiratory Journal Print ISSN 0903-1936

Online ISSN 1399-3003 
Tyr113His polymorphism with a recessive odds ratio (OR) of 3.5 [14], whereas subsequent studies failed to confirm this [15, 16]. Large studies have also linked the same SNP in this gene to lung cancer, using the powerful incident cases design or very large pooled studies [17-19].

TNF is a multifunctional pro-inflammatory cytokine. Several regulatory polymorphisms have been investigated (online Mendelian inheritance in man (OMIM):191160) [19]. An initial study determined a very high OR of $>10$ for an association with COPD [20], whereas subsequent studies found smaller ORs [21, 22]. ADRB2 is of importance because $\beta_{2}$-receptor agonists are widely used for the treatment of obstructive lung disease. Two coding polymorphisms of functional significance have been extensively studied from a pharmacogenetic perspective (OMIM:109690), but only one previous study has assessed its role in the susceptibility of COPD [23].

\section{MATERIALS AND METHODS Populations and screening}

Potential cases and controls were searched for from a hospital population and a general population cohort study. From computerised hospital records at Haukeland University Hospital (Bergen, Norway), all living patients with a recorded COPD diagnosis during the period 1997-2002, without exclusion criteria, were identified. Potential controls and cases were also identified from an ongoing general adult population cohort study in the city of Bergen, and surrounding areas, which had been running since 1985 [24]. From January to June 2003, 1,478 persons were invited in random order from the combined database (551 patients, 910 cohort participants, 17 volunteers). Of these, 1,111 attended a clinical examination that included lung function tests, questionnaires and peripheral blood samples. Attendance rates were $75 \%$ in both the patient registry and cohort sample. From patient registry attendees, $48 \%$ were selected as cases and $10 \%$ as controls. From cohort participant attendees, $6 \%$ were selected as cases and $29 \%$ as controls. Of the attendees, 248 were assigned case status and 244 control status, according to the inclusion and exclusion criteria.

\section{Inclusion and exclusion criteria}

Common criteria for both cases and control were: 1) selfreported Caucasian; 2) aged $\geqslant 40$ yrs; 3) current or former smokers with at least 2.5 pack-yrs smoking history; 4) no severe $\alpha_{1}$-antitrypsin deficiency (ZZ, Z null, null-null or S-Z); and 5) no previous chronic lung disease, dementia, HIV, hepatitis $\mathrm{B}$ or $\mathrm{C}$. Cases were required to have COPD at stage II or worse [1], i.e. a post-bronchodilator forced expiratory volume in one second (FEV1) $>80 \%$ of predicted value and FEV1 to forced vital capacity (FVC) ratio $>70 \%$. Controls were required to have an FEV1 of $>80 \%$ pred and $\mathrm{FEV} 1 / \mathrm{FVC}$ ratio $>70 \%$. There was no phenotyping related to asthma.

Spirometry was performed according to American Thoracic Society standards after bronchodilation with $400 \mu \mathrm{g}$ of salbutamol via a metered-dose inhaler and a spacer chamber. Subjects were assessed at least 6 weeks after any respiratory infection, but were not asked to withhold regular medication.

\section{Genotyping procedures}

DNA was extracted from frozen whole blood. SNP genotyping was performed with the Assay-on-Demand ${ }^{\mathrm{TM}}$ and
Assay-by-Design ${ }^{\mathrm{TM}}$ system (Applied Biosystems) based on the Taqman PCR assay using minor groove-binder probes. Assays were run in a 96-well format according to the manufacturer's instructions. End-point allelic discrimination was performed on an ABI Prism 7900HT unit (Applied Biosystems). Details of primers and probes and dbSNP identification of the SNPs can be found as online supplementary material.

\section{Statistical analysis and meta-analysis}

In the case-control study, statistical comparisons between groups used t-tests for continuous variables and Fisher's exact Chi-squared tests for binary variables. ORs for each SNP were computed and adjusted ORs were computed with logistic regression, adjusting for age, sex and pack-years. A systematic literature review was performed to identify previous casecontrol studies of genetic susceptibility to COPD using computer-based searches and scanning of the reference lists of all identified relevant studies and review articles. The computer search was performed using MEDLINE and EMBASE. The search criteria included information up to June 2004.

Studies without Hardy-Weinberg equilibrium (HWE) for a given SNP were excluded from that analysis. Some studies had more than one control group as well as different phenotypes. A nonconservative pooled estimate was first calculated using all phenotype-genotype associations, thus counting some studies and some cases more than once. Further pooled estimates in subgroups were made in various combinations of phenotype, ethnicity and control group type.

Meta-analysis was performed with inverse variance weighting, i.e. the logarithm of the OR from each study was weighted by a function of its variance and pooled. Random-effect models were used that allowed for between-study heterogeneity [25].

\section{Regulatory approval and informed consent}

The case-control study was approved by the Ethical Review Board of Western Norway, the Norwegian Data Inspectorate and the Norwegian Department of Health. Written informed consent was obtained from all participants.

\section{RESULTS}

\section{Case-control study}

Table 1 shows the demographic characteristics and lung function measurements of cases and controls. Controls were slightly younger than cases, more were female and more were current smokers. Controls had a slight reduction in lung function measurements, whereas cases, as expected, had a much larger reduction in lung function, typical of a clinical COPD population. The absolute levels of lung function had the same standard deviations, but the per cent predicted had higher standard deviations in cases than controls. This reflects the heterogeneity in the COPD group. Cases had been exposed substantially more to tobacco smoke, as evidenced by the difference in 10 pack-yrs between the cases and controls.

Genotype and allele frequencies by case-control status are given in table 2. There was no deviation from HWE in controls. The EPHX1 Tyr113His (fast) polymorphism was significantly associated with COPD ( $\mathrm{p}=0.02$ under a codominant model). Adjustment for age, sex and pack-years did not change the OR, 


\begin{tabular}{lccc} 
TABLE 1 & $\begin{array}{l}\text { Demographic characteristics and lung function } \\
\text { measurements in this study }\end{array}$ \\
& Controls & Cases & p-value $^{\#}$ \\
\hline & 248 & 244 & \\
Subjects n & $54.9 \pm 10.2$ & $58.4 \pm 8.2$ & $<0.001$ \\
Age yrs & 51.6 & 59.0 & 0.10 \\
Males \% & 57.3 & 48.8 & 0.07 \\
Current smoker \% & $19.1 \pm 13.4$ & $28.8 \pm 15.1$ & $<0.001$ \\
Pack-yrs of smoking & $3.30 \pm 0.75$ & $1.69 \pm 0.75$ & $<0.001$ \\
FEV1 L & $4.15 \pm 0.93$ & $3.16 \pm 1.03$ & $<0.001$ \\
FVC L & $95.4 \pm 10.0$ & $50.0 \pm 18.4$ & $<0.001$ \\
FEV $\mathbf{1} \%$ pred & $97.9 \pm 10.1$ & $76.0 \pm 17.1$ & $<0.001$ \\
FVC \% pred & $0.80 \pm 0.04$ & $0.52 \pm 0.13$ & $<0.001$ \\
FEV1/FVC ratio & & & \\
\hline
\end{tabular}

Data are presented as mean $\pm S D_{\text {, }}$ unless otherwise stated. FEV 1 : forced expiratory volume in one second; FVC: forced expiratory volume; \% pred: per cent predicted. ${ }^{\#}$ : difference between cases and controls.

but the $\mathrm{p}$-value was somewhat attenuated $(\mathrm{p}=0.06)$. The allele frequencies were similar among cases and controls ( 0.31 and 0.30 , respectively), but the frequency of minor allele homozygotes was clearly reduced in COPD cases. This explains the reduced variant homozygote risk $(\mathrm{OR}=0.5)$. ORs for other SNPs or models were in the range of $0.81-1.26$. Polymorphisms in the TNF-376 and TNF-238 SNPs were rare.

\section{Meta-analysis}

The present authors' literature search identified 49 publications, of which 17 publications concerned the SNPs in question [14-16, 20-22, 26-35]. During the literature search, 14 additional genes used in COPD genetic association studies were found, but with mostly single studies (genes: DEFB1, IL1B, IL13, IL4R, IL13RA1, GSTT1, CYP1A1, CYP2E1, LTA, HP, RHD, TF, FUT2, FUT7). A recent publication identified after the literature search was not included in the metaanalysis because it did not give sufficient data [36]. Two studies were excluded, one because it only reported allele frequencies [37], and another because it was in Chinese. One study had probably reversed its assignment of genotypes of the TNF-308 SNP [20], which was corrected in the meta-analysis.

Details of selection criteria for previous studies can be found as online supplementary material. All studies were prevalent case based. Spirometric COPD was almost universally taken as FEV1/FVC ratio $>0.7$ and FEV1 $<80 \%$ or worse. In contrast, the case and control recruitment and selection criteria were quite heterogeneous. Only six studies performed spirometries in control subjects. Only five studies required a smoking history in cases or controls, and others had various other exclusion criteria. All studies except the one by KUCUKAYCAN et al. [29] used the restriction fragment length polymorphism (RFLP) technique for genotyping.

Three SNPs examined by the present authors had been included in more than one previous study. Figure 1 shows ORs from all the studies. In the controls, as many as seven out of 15 individual studies had HWE absent for one or more SNPs. Meta-analytical estimates are given in table 3 with a

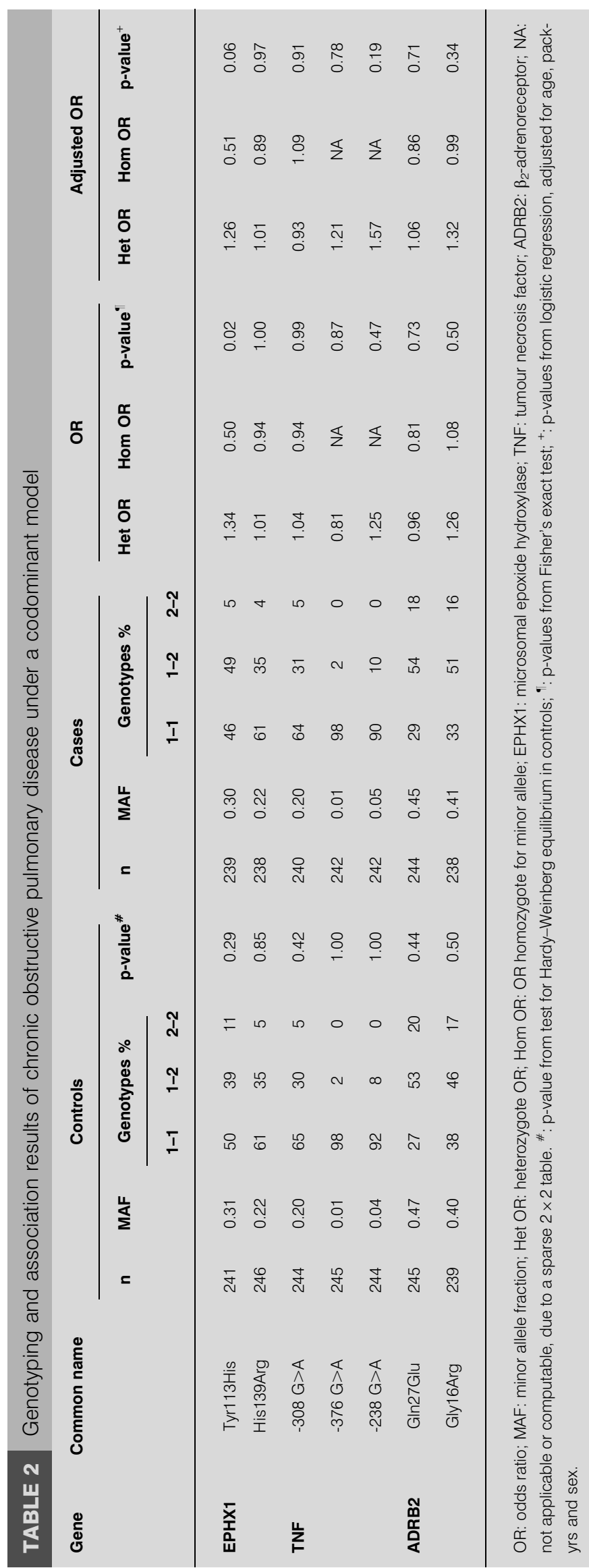




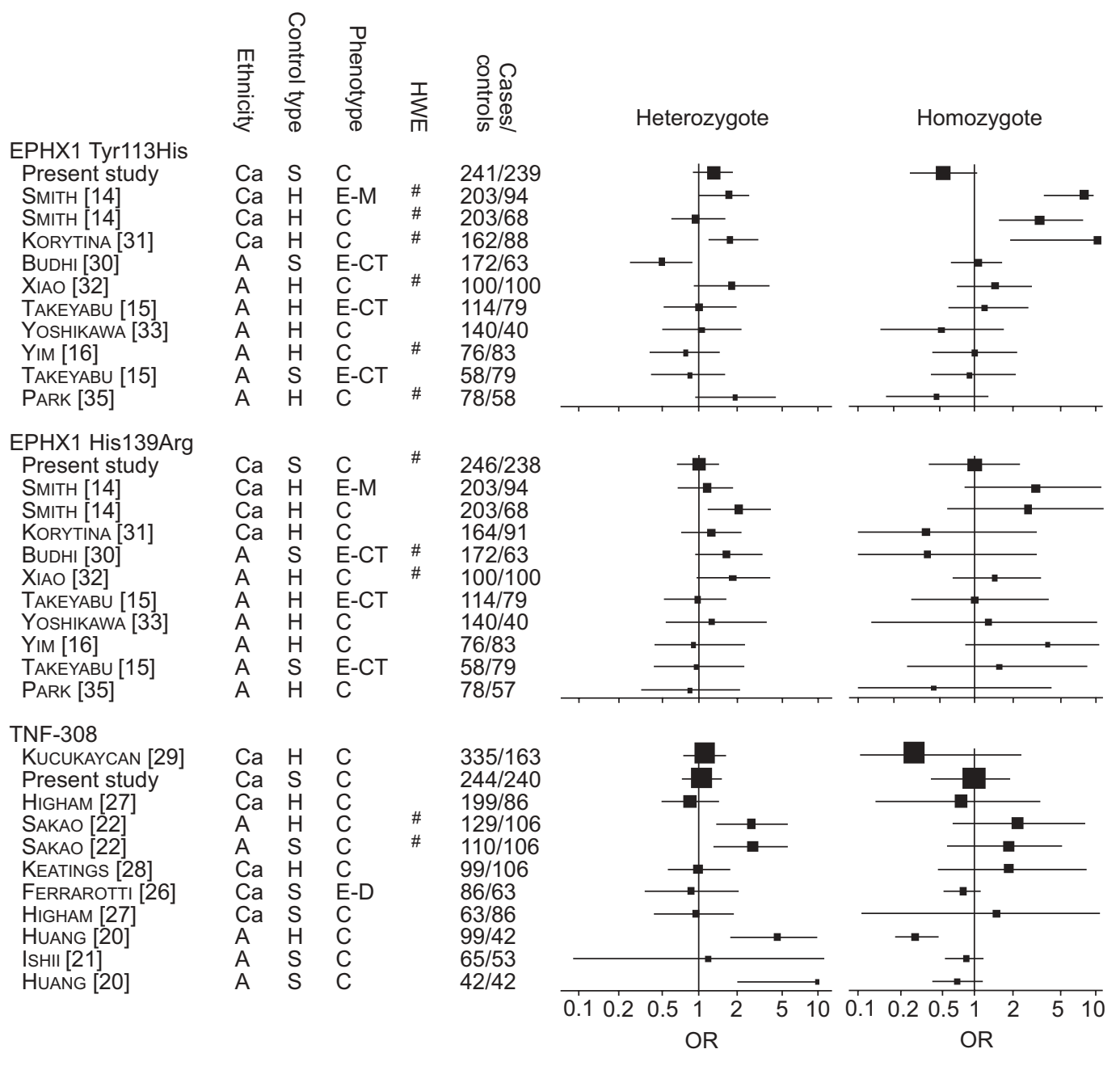

FIGURE 1. Odds ratios (OR) from previous studies of three single nucleotide polymorphisms (microsomal epoxide hydroxylase (EPHX1) Tyr113His, EPHX1 His139Arg and tumour necrosis factor (TNF)-308, respectively) and their association with spirometric chronic obstructive pulmonary disease (COPD) or emphysema, listed in decreasing order of sample size. Effect estimates are shown under a codominant model for heterozygotes and homozygote for the minor allele. Marker size is proportional to study size. Ca: Caucasian; A: Asian; S: smokers; H: healthy; C: COPD; E-M: emphysema based on morphology; E-CT: emphysema based on computed tomography scan; E-D: emphysema based on diffusing capacity for carbon monoxide; HWE: Hardy-Weinberg equilibrium. \#: absence of HWE.

nonconservative estimate across all associations, and then stratified by various more or less interesting combinations of phenotype and ethnic group.

There were eight previous studies of the EPHX1 Tyr113His polymorphism, from which six associations were excluded due to the absence of HWE in controls [14, 16, 31, 32, 35]. Five associations remained from the current study and three previous studies $[15,30,33]$. All of the three previous studies were of Asian ethnicity. Meta-analysis of all associations did not suggest an association. Stratifying by phenotype showed a significant association of COPD with homozygotes for the minor allele (OR 0.5; $\mathrm{p}=0.03$ ) based on two studies, the present study and the relatively small study by YOSHIKAWA et al. [33]. There was no association between Tyr113His and emphysema.

There were eight previous studies of the EPHX1 His139Arg polymorphism, from which two studies were excluded due to absence of HWE [30, 32]. Nine associations remained from the current study and seven previous studies [14-16, 31-33, 35]. There was no association overall or in analyses stratified by phenotype, control group type or ethnicity.

There were seven previous studies of the TNF-308 polymorphism, of which one was excluded due to the absence of HWE [22]. Nine associations from seven studies were available [21, 26-29], of which one was emphysema [26]. There was no association overall and no association in various stratifications on control group and ethnicity.

The study by KUCUKAYCAN et al. [29] of two other TNF polymorphisms had an absence of HWE in TNF-376. For TNF-238, a meta-analysis (575 controls, 476 cases) gave a heterozygote OR of $1.28(\mathrm{p}=0.30)$. In the study by Ho et al. [23] of ADRB2, polymorphisms had an absence of HWE in Gln27Glu. For Arg16Gly, there was no effect in a meta-analysis $(\mathrm{OR}=2.1 ; \mathrm{p}=0.21)$.

Data regarding differences in genotype prevalence by ethnicity from the dbSNP database are as follows [38]: 1) no difference 


\begin{tabular}{|c|c|c|c|c|c|c|c|c|c|c|c|}
\hline TABLE 3 & $\begin{array}{l}\text { Meta-an } \\
\text { spirome } \\
\text { homozy }\end{array}$ & $\begin{array}{l}\text { sis from } \\
\text { chronic } \\
\text { e for the }\end{array}$ & $\begin{array}{l}\text { vious st } \\
\text { structive } \\
\text { nor allele }\end{array}$ & $\begin{array}{l}\text { es of thre } \\
\text { almonary }\end{array}$ & $\begin{array}{l}\text { single } \\
\text { sease }(4\end{array}$ & $\begin{array}{l}\text { ucleotid } \\
\text { OPD) }\end{array}$ & $\begin{array}{l}\text { olymorph } \\
\text { r a codo }\end{array}$ & $\begin{array}{l}\text { (SNF } \\
\text { ht mc }\end{array}$ & $\begin{array}{l}\text { nd their } \\
\text { for heter }\end{array}$ & $\begin{array}{l}\text { ociati } \\
\text { gotes }\end{array}$ & $\begin{array}{l}\text { with } \\
\text { d }\end{array}$ \\
\hline \multicolumn{5}{|c|}{ Study selection criteria } & \multicolumn{7}{|c|}{ Results } \\
\hline \multirow[t]{2}{*}{ SNP } & \multirow[t]{2}{*}{ Pheno. } & \multirow[t]{2}{*}{ Ethnicity } & \multirow{2}{*}{$\begin{array}{l}\text { Control } \\
\text { groups }\end{array}$} & \multirow{2}{*}{$\begin{array}{c}\text { Multiple } \\
\text { control } \\
\text { groups }^{\#}\end{array}$} & \multirow[t]{2}{*}{ Assoc. } & \multirow[t]{2}{*}{ Cases } & \multirow[t]{2}{*}{ Controls } & \multicolumn{2}{|c|}{ Heterozygote } & \multicolumn{2}{|c|}{ Homozygote } \\
\hline & & & & & & & & OR & $\mathrm{p}$-value & OR & p-value \\
\hline \multicolumn{12}{|l|}{ EPHX1 } \\
\hline \multirow[t]{3}{*}{ Tyr113His } & B & B & B & Yes & 5 & 500 & 725 & 0.95 & 0.80 & 0.79 & 0.20 \\
\hline & C & B & B & No & 2 & 279 & 381 & 1.28 & 0.15 & 0.50 & 0.03 \\
\hline & E & A & S & No & 2 & 142 & 230 & 0.61 & 0.07 & 0.93 & 0.80 \\
\hline \multicolumn{12}{|l|}{ EPHX1 } \\
\hline \multirow[t]{5}{*}{ His139Arg } & B & B & B & Yes & 9 & 829 & 1282 & 1.15 & 0.18 & 1.30 & 0.32 \\
\hline & C & B & B & No & 6 & 577 & 907 & 1.2 & 0.21 & 1.17 & 0.61 \\
\hline & C & $\mathrm{Ca}$ & B & No & 3 & 397 & 613 & 1.36 & 0.19 & 1.00 & 0.99 \\
\hline & C & A & $\mathrm{H}$ & No & 3 & 180 & 294 & 0.97 & 0.90 & 1.58 & 0.48 \\
\hline & E & B & B & Yes & 3 & 252 & 375 & 1.06 & 0.75 & 1.55 & 0.32 \\
\hline \multirow[t]{6}{*}{ TNF G-308A } & B & B & B & Yes & 9 & 881 & 1232 & 1.25 & 0.22 & 0.67 & 0.02 \\
\hline & C & B & B & Yes & 8 & 818 & 1146 & 1.31 & 0.17 & 0.66 & 0.07 \\
\hline & C & B & S & No & 5 & 527 & 513 & 1.23 & 0.43 & 0.81 & 0.12 \\
\hline & C & B & $\mathrm{H}$ & No & 3 & 291 & 633 & 1.49 & 0.31 & 0.32 & $<0.001$ \\
\hline & C & A & B & Yes & 3 & 137 & 206 & 5.05 & $<0.001$ & 0.55 & 0.06 \\
\hline & C & $\mathrm{Ca}$ & B & Yes & 5 & 681 & 940 & 1.01 & 0.90 & 0.96 & 0.91 \\
\hline
\end{tabular}

Data are presented as n, unless otherwise stated. Pheno.: phenotype; Assoc.: association; OR: odds ratio; B: both; C: COPD; E: emphysema; A: Asian; Ca: Caucasian; S: smoking; H: healthy. "\#: some studies had multiple control groups; if "yes", each association between cases and different control groups was used, thus counting cases twice.

for EPHX1 Tyr113His (major allele frequency range (MAF) 63$64 \%$ ); 2) no data available for EPHX1 His139Arg; and 3) some differences for TNF-308 (MAF range 83-97\%).

\section{DISCUSSION}

In a single genetic association study and meta-analysis, a significant effect of the EPHX1 Tyr113His polymorphism on susceptibility to COPD was found. The effects of six other SNPs were small and few reached conventional levels of significance. Significant heterogeneity of phenotyping and absence of HWE was found in $40 \%$ of previous studies.

In the present context, absence of HWE is probably a genotyping error [39]. The RFLP technique is known to be biased away from heterozygotes [40]. This was confirmed in a recent paper comparing TaqMan genotyping and RFLP for the EPHX1 Tyr113His SNP [17].

To the current authors' knowledge, this is the first metaanalysis of non- $\alpha_{1}$-antitrypsin genetic associations in COPD.

The inability to replicate genetic associations has led to increasing scepticism about the value of genetic association studies [10, 11, 41]. There are many reasons for nonreplication apart from statistical error. Prominent sources of heterogeneity include: ethnic heterogeneity; variable phenotypes and different cut-off points; selection bias due to convenience controls; and use of prevalent cases leading to survival bias. Surprisingly, genotyping error was also quite common. All of these sources of error were present in the collection of previous studies identified. There is no substitute for large studies with well-defined phenotypes. Meta-analysis remains an essential tool for summarising previous studies until such large studies become available [11].

Insufficient statistical power remains a probable large contributor to lack of replication. The present study had findings that were somewhat statistically weak in relation to the number of statistical tests. Conversely, it was a replication of previous associations, thus increasing the prior probability of association. A recent meta-analysis of many different genetic associations found that a sizeable fraction of initial genetic associations were in fact replicated [41].

A recent review and replication attempt of previous COPD genetic association studies was more comprehensive in terms of genes tested than the current study [36]. It was a large casecontrol and family-based study, but only included severe COPD (FEV1 <25\%). Only two of the 19 SNPs tested were also studied in the present study (EPHX1 and TNF). The results were disappointing: no associations were convincingly replicated across both study designs. However, in the case-control portion, there was an association with EPHX1, but only with the His139Arg SNP $(p=0.02)$. The observed differences between family-based and case-control associations could be biological, due to different phenotypes. The present authors observed an association between Tyr113His, whereas HERSH et al. [36] found an association between His139Arg. The bulk of the evidence in other smoking-related diseases suggests that Tyr113His is the causal polymorphism. 
The weakest link in all previous studies of COPD, including the present study, is smoking. COPD is a prototypical model of a disease with a major gene-by-environment interaction. Very limited information was available on smoking histories in previous studies. In the current case-control study, the packyear requirement was low and there was an imbalance in packyears, age and sex between the case and control groups. Hence, a number of the controls could develop COPD in the future. Similarly, many previous studies used young controls that could develop COPD in the future. It is important to realise that bias is a question of degree and is not insurmountable. These biases would in fact tend to dilute the true effect of any causal polymorphism. The association of EPHX1 Tyr113His was unchanged after adjustment for age, pack-years and sex, which suggests that this is a robust effect in the current population.

The ideal COPD genetic association study would be large, longitudinal, and use the cumulative reduction in lung function in relation to cumulative smoking as a main outcome measure. Such a study would take decades to complete, and require tens of thousands of subjects. For genetic association studies of most chronic diseases, the inevitable environmental component is hardly known at all. The rationale is, and will probably remain for a substantial period of time, that the genetic contribution to disease will give a sufficiently strong signal even in the case of gene-environment interactions. However, in the future, attention to study design issues could substantially enhance the chance of finding causal polymorphisms for COPD.

The functional role of EPHX1 and its polymorphisms has not been as well studied as some other genes, but the polymorphisms appear to be functional [12, 13]. Biologically, a high enzyme activity might both be pro-carcinogenic or anticarcinogenic, depending on the specific carcinogen [42]. This might explain the paradox that the Tyr113His polymorphism increases the risk of colorectal cancer in smokers [42], but protects from lung cancer $[17,18]$.

Several large association studies of lung cancer susceptibility have found results that are very similar to the current results, i.e. a protective effect for homozygotes of the Tyr113His SNP with an OR of 0.4 , but no effect of the His139Arg polymorphism. These studies were larger and stronger than the present study because they studied incident lung cancer, and, thus, were not susceptible to early death. The present study can in fact not discern between a protective effect of Tyr113His towards COPD development, or a harmful effect due to early death of COPD homozygotes. In summary, there is quite a lot of evidence to implicate the Tyr113His polymorphism in smoking-related diseases, both in functional and association studies.

Regulatory polymorphisms in TNF have been extensively studied across diseases and populations, but the results only seem consistent for infectious diseases [19]. The TNF-308 polymorphism is perhaps not even functional, while the role of other TNF polymorphisms remains unclear [19]. The one significant association found in the meta-analysis was between COPD and healthy controls, while there was no association with smoking controls.
The ADRB2 polymorphisms have been studied in asthma pharmacogenetics with interesting results as well as susceptibility to obesity (OMIM), whereas their relationship with susceptibility to asthma has not been confirmed. The evidence for any association with COPD is weak, since the one previous small study [23] possibly had a genotyping error in one polymorphism and the meta-analysis of the other was insignificant. The present study was adequately powered to find a moderate genetic risk, so it can be concluded that there was no meaningful effect of the ADRB2 polymorphism.

In conclusion, the microsomal epoxide hydroxylase Tyr113His polymorphism is associated with a protection from chronic obstructive pulmonary disease, whereas six other candidate single nucleotide polymorphisms were not. Future genetic association studies of chronic obstructive pulmonary disease should account for possible effects of survival bias, and be rigorous in genotyping quality control.

\section{REFERENCES}

1 Pauwels RA, Buist AS, Calverley PM, Jenkins CR, Hurd SS. Global strategy for the diagnosis, management, and prevention of chronic obstructive pulmonary disease. NHLBI/WHO Global Initiative for Chronic Obstructive Lung Disease (GOLD) Workshop summary. Am J Respir Crit Care Med 2001; 163: 1256-1276.

2 Burrows B, Knudson RJ, Cline MG, Lebowitz MD. Quantitative relationships between cigarette smoking and ventilatory function. Am Rev Respir Dis 1977; 115: 195-205.

3 Silverman EK. Genetic epidemiology of COPD. Chest 2002; 121: 1S-6S.

4 Sandford AJ, Silverman EK. Chronic obstructive pulmonary disease. 1: Susceptibility factors for COPD the genotype-environment interaction. Thorax 2002; 57: 736-741.

5 Wilk JB, DeStefano AL, Arnett DK, et al. A genome-wide scan of pulmonary function measures in the national heart, lung, and blood institute family heart study. Am J Respir Crit Care Med 2003; 167: 1528-1533.

6 Palmer LJ, Celedon JC, Chapman HA, Speizer FE, Weiss ST, Silverman EK. Genome-wide linkage analysis of bronchodilator responsiveness and post-bronchodilator spirometric phenotypes in chronic obstructive pulmonary disease. Hum Mol Genet 2003; 12: 1199-1210.

7 Silverman EK, Mosley JD, Palmer LJ, et al. Genome-wide linkage analysis of severe, early-onset chronic obstructive pulmonary disease: airflow obstruction and chronic bronchitis phenotypes. Hum Mol Genet 2002; 11: 623-632.

8 Lomas DA, Silverman EK. The genetics of chronic obstructive pulmonary disease. Respir Res 2001; 2: 20-26.

9 Molfino NA. Genetics of COPD. Chest 2004; 125: 1929-1940.

10 Ioannidis JP, Trikalinos TA, Ntzani EE, ContopoulosIoannidis DG. Genetic associations in large versus small studies: an empirical assessment. Lancet 2003; 361: 567-571.

11 Salanti G, Sanderson S, Higgins JP. Obstacles and opportunities in meta-analysis of genetic association studies. Genet Med 2005; 7: 13-20.

12 Maekawa K, Itoda M, Hanioka N, et al. Non-synonymous single nucleotide alterations in the microsomal epoxide 
hydrolase gene and their functional effects. Xenobiotica 2003; 33: 277-287.

13 Omiecinski CJ, Hassett C, Hosagrahara V. Epoxide hydrolase - polymorphism and role in toxicology. Toxicol Lett 2000; 112-113: 365-370.

14 Smith CAD, Harrison DJ. Association between polymorphism in gene for microsomal epoxide hydrolase and susceptibility to emphysema. Lancet 1997; 350: 630-633.

15 Takeyabu K, Yamaguchi E, Suzuki I, Nishimura M, Hizawa N, Kamakami Y. Gene polymorphism for microsomal epoxide hydrolase and susceptibility to emphysema in a Japanese population. Eur Respir J 2000; 15: 891-894.

16 Yim JJ, Park GY, Lee CT, et al. Genetic susceptibility to chronic obstructive pulmonary disease in Koreans: combined analysis of polymorphic genotypes for microsomal epoxide hydrolase and glutathione $S$-transferase M1 and T1. Thorax 2000; 55: 121-125.

17 Gsur A, Zidek T, Schnattinger K, et al. Association of microsomal epoxide hydrolase polymorphisms and lung cancer risk. Br J Cancer 2003; 89: 702-706.

18 Lee WJ, Brennan P, Boffetta P, et al. Microsomal epoxide hydrolase polymorphisms and lung cancer risk: a quantitative review. Biomarkers 2002; 7: 230-241.

19 Bayley JP, Ottenhoff TH, Verweij CL. Is there a future for TNF promoter polymorphisms? Genes Immun 2004; 5: 315-329.

20 Huang SL, Su CH, Chang SC. Tumor necrosis factor-alpha gene polymorphism in chronic bronchitis. Am J Respir Crit Care Med 1997; 156: 1436-1439.

21 Ishii T, Matsuse T, Teramoto S, et al. Neither IL-1beta, IL-1 receptor antagonist, nor TNF-alpha polymorphisms are associated with susceptibility to COPD. Respir Med 2000; 94: 847-851.

22 Sakao S, Tatsumi K, Igari H, Shino Y, Shirasawa H, Kuriyama T. Association of tumor necrosis factor alpha gene promoter polymorphism with the presence of chronic obstructive pulmonary disease. Am J Respir Crit Care Med 2001; 163: 420-422.

23 Ho LI, Harn HJ, Chen CJ, Tsai NM. Polymorphism of the beta(2)-adrenoceptor in COPD in Chinese subjects. Chest 2001; 120: 1493-1499.

24 Eagan TM, Bakke P, Eide GE, Gulsvik A. Incidence of asthma and respiratory symptoms by sex, age and smoking in a community study. Eur Respir J 2002; 19: 599-605.

25 Sutton AJ. Random effects methods for combining study estimates. Methods for meta-analysis in medical research. Chichester, UK, J Wiley and Sons, 2000; pp. 73-86.

26 Ferrarotti I, Zorzetto M, Beccaria M, et al. Tumour necrosis factor family genes in a phenotype of COPD associated with emphysema. Eur Respir J 2003; 21: 444-449.

27 Higham MA, Pride NB, Alikhan A, Morrell NW. Tumour necrosis factor-alpha gene promoter polymorphism in chronic obstructive pulmonary disease. Eur Respir J 2000; 15: 281-284.

28 Keatings VM, Cave SJ, Henry MJ, et al. A polymorphism in the tumor necrosis factor-alpha gene promoter region may predispose to a poor prognosis in COPD. Chest 2000; 118 : 971-975.

29 Kucukaycan M, Van Krugten MV, Pennings $H$, et al. Tumor necrosis factor $\alpha+489 \mathrm{G} / \mathrm{A}$ gene polymorphism is associated with chronic obstructive pulmonary disease. Respir Res 2002; 3: 29.

30 Budhi A, Hiyama K, Isobe T, et al. Genetic susceptibility for emphysematous changes of the lung in Japanese. Int J Mol Med 2003; 11: 321-329.

31 Korytina GF, Ianbaeva DG, Viktorova TV. Polymorphisms of the cytochrome P450 (CYP1A1,CYP2E1) and microsomal epoxide hydrolase ( $\mathrm{mEPHX}$ ) genes in cystic fibrosis and chronic respiratory disease. Mol Biol (Mosk) 2003; 37: 784-792.

32 Xiao D, Wang C, Du MJ, et al. Relationship between polymorphisms of genes encoding microsomal epoxide hydrolase and glutathione S-transferase P1 and chronic obstructive pulmonary disease. Chin Med J (Engl) 2004; 117: 661-667.

33 Yoshikawa M, Hiyama K, Ishioka S, Maeda H, Maeda A, Yamakido M. Microsomal epoxide hydrolase genotypes and chronic obstructive pulmonary disease in Japanese. Int J Mol Med 2000; 5: 49-53.

34 Zhang R, Zhang A, He Q, Lu B. Microsomal epoxide hydrolase gene polymorphism and susceptibility to chronic obstructive pulmonary disease in Han nationality of North China. Zhonghua Nei Ke Za Zhi 2002; 41: 11-14.

35 Park SS, Kim EJ. Genetic polymorphism of epoxide hydrolase and GSTM1 in chronic obstructive pulmonary disease. Tuberc Respir Dis 2003; 55: 88-97.

36 Hersh CP, DeMeo DL, Lange C, et al. Attempted replication of reported chronic obstructive pulmonary disease candidate gene associations. Am J Respir Cell Mol Biol 2005; 33: 71-78.

37 Patuzzo C, Gile LS, Zorzetto M, et al. Tumor necrosis factor gene complex in COPD and disseminated bronchiectasis. Chest 2000; 117: 1353-1358.

38 Sherry ST, Ward MH, Kholodov M, et al. dbSNP: the NCBI database of genetic variation. Nucleic Acids Res 2001; 29: 308-311.

39 Hosking L, Lumsden S, Lewis K, et al. Detection of genotyping errors by Hardy-Weinberg equilibrium testing. Eur J Hum Genet 2004; 12: 395-399.

40 Wu YY, Delgado R, Costello R, Sunderland T, Dukoff R, Csako G. Quantitative assessment of apolipoprotein E genotypes by image analysis of PCR-RFLP fragments. Clin Chim Acta 2000; 293: 213-221.

41 Lohmueller KE, Pearce CL, Pike M, Lander ES, Hirschhorn JN. Meta-analysis of genetic association studies supports a contribution of common variants to susceptibility to common disease. Nat Genet 2003; 33: 177-182.

42 Huang WY, Chattee N, Chanock S, et al. Microsomal epoxide hydrolase polymorphisms and risk for advanced colorectal adenoma. Cancer Epidemiol Biomarkers Prev 2005; 14: 152-157. 